Review Article

\title{
Current Review on Molecular and Phenotypic Mechanism of Bacterial Resistance to Antibiotic
}

\author{
Mesele Admassie \\ Department of Microbial, Cellular and Molecular Biology, Addis Ababa University, Addis Ababa, Ethiopia \\ Email address: \\ mesele2006@gmail.com
}

To cite this article:

Mesele Admassie. Current Review on Molecular and Phenotypic Mechanism of Bacterial Resistance to Antibiotic. Science Journal of Clinical Medicine. Vol. 7, No. 2, 2018, pp. 13-19. doi: 10.11648/j.sjcm.20180702.11

Received: March 9, 2018; Accepted: April 2, 2018; Published: May 8, 2018

\begin{abstract}
Antibiotic-resistant bacteria that are difficult to treat are becoming increasingly common and are causing a global health crisis. molecular mechanism bacterial resistance of antibiotic is, necessary in order to find ways to avoid conditions that promote disobedient pathogens. The objectives of this seminar paper are to review the genetics of molecular mechanism of antibiotic resistance and to describe different molecular intrinsic, acquired, circumstantial resistance; genetics of antibiotic resistance and phenotypic drug resistance those specified by mechanisms confer antibiotic resistance. These drug resistance mechanisms allow bacteria that harbor these mechanisms to survive, or even to actively grow, in the presence of a given anti-microbial agent. Furthermore, certain bacterial variants have evolved mechanisms to resist multiple drugs, making such variants recalcitrant to chemotherapy against such bacterial strains that are the causative agents of infection in patients. Knowing the molecular mechanisms of antibiotic resistance is essential for developing new approaches to overcome and prevent drug resistance problems. Studding of development and mechanisms of resistance should be known at an early stage of drug development and strategies to improve the delivery or to enhancing the accessibility of antibiotics to their sites of action should be known.
\end{abstract}

Keywords: Antibiotic Resistant, Molecular Mechanism, Bacteria, Intrinsic Resistance

\section{Introduction}

Bacteria that are causative agents of infectious disease represent a serious public health concern globally. The history of antibiotic resistance coincides with the history of antibiotics themselves. Ironically, penicillin resistance was discovered even before penicillin was put to clinical use. The first antibiotic resistance mechanism reported in the literature was the production of Penicillinase by pathogenic Escherichia coli. Bacteria may be intrinsically resistant to antibacterial agents or acquire resistance by mutation or acquisition of resistance determinants. Bacterial resistance is closely associated with the use of antimicrobial agents in clinical practice. Prolonged therapy with antibiotics can lead to the development of resistance in a microorganism that initially is sensitive to antibiotics, but later it can adapt gradually and develop resistance to antibiotics. The emergence of a phenotype resistant to antimicrobial agents depends on various factors of a host: degree of resistance expression, capability of a microorganism to tolerate resistance mechanism, initial colonization site, and other factors. When resistance determinants are on plasmids, they will spread quickly within the genus and even unrelated bacterial genera. When resistance is associated with genes on chromosomes, resistant microorganisms will spread more slowly [1]. From the 1950s to the late $1990 \mathrm{~s}$, concerns about anti-microbial resistance were alleviated by the development of a steady stream of new antimicrobials which has now virtually ceased [2]. Common gram-negative bacteria and other organisms have genetic mechanisms of developing resistance that can evade the effects of antibiotics. These include genes that enable organisms to produce enzymes such as extended spectrum beta lactamases and various carbapenemases, efflux pump mechanisms and plasmids carrying resistance genes. Recent reports of the developmentofcolistin resistance in Escherichia coli mediated by a plasmid carrying themobilized colistin resistance (mcr-1) gene have caused great concern. Colistin is 
generally considered to be one of the antibiotics of last resort. The broader global implications of Antimicrobial resistance (AMR) are underscored by the fact that several highly resistant bacteria isolates were first reported in Asia and linked to the animal use of antibiotic [3].

(AMR) is a significant and increasing threat to public health globally. It is estimated that in the US and Europe alone, antimicrobial-resistant infections currently cause at least 50,000 deaths per year with hundreds of thousands more dying in other areas of the world. If we are unable to slow the acceleration of AMR, future consequences will be worse still. The Review on antimicrobial resistance has estimated that 10 million people a year could be dying as a result of AMR by 2050. where 40 per cent of the population die prematurely from infections we cannot treat [4].

The over-and miss-use of antibiotics are the main drivers in the selection of resistant bacteria. The successful development of new antibiotics and novel treatments such as monoclonal antibodies, antitoxins, probiotics and bacteriophage therapies have to be accompanied by a commitment to use them with great care to ensure their long-term effectiveness [5]. Antibiotic-resistant bacteria that are difficult to treat are becoming increasingly common and are causing a global health crisis [6].

Review of this molecular mechanism bacterial resistance of antibiotic is, therefore of a necessary in order to find ways to avoid conditions that promote such disobedient pathogens. Molecular, biochemical, physiological and structural analyses of bacterial multiple drug resistance mechanisms will promote their putative modulation [5].

The objective of this seminar paper is to review the molecular mechanism of antibiotic resistance and to describe different mechanism such as: intrinsic, acquired, and circumstantial; genetics, and phenotypic drug resistance; these helps to develop screening and control strategiesthat are needed to reduce the spread of resistant bacteria and their evolution.

Table 1.The bacterial mechanisms of antibiotic resistance [7].

\begin{tabular}{|c|c|c|c|}
\hline Basis ofresistance & Mechanism & Bacterial proteins/targetsresponsible & Antibiotic targets \\
\hline \multirow{8}{*}{ Enzyme } & \multirow{3}{*}{ Hydrolysis } & $\beta$-lactamases & $\beta$-lactamases \\
\hline & & Esterase & Macrolide \\
\hline & & C-P lyase complex & Fosfomyci \\
\hline & \multirow{5}{*}{ Group transfer } & Acetyltransferase & $\begin{array}{l}\text { Streptogramins, amino } \\
\text { glycosides, chloramphenicol }\end{array}$ \\
\hline & & Phosphotransferase & Aminoglycosides, macrolides \\
\hline & & Nucleotidyltransferase & $\begin{array}{l}\text { Lincomycin, clindamycin, } \\
\text { aminoglycosides }\end{array}$ \\
\hline & & Ribosyltransferase & Rifampin \\
\hline & & Thioltransferase & Fosfomycin \\
\hline \multirow{7}{*}{ Target modification } & Redox process & TetX & Tetracyclines \\
\hline & \multirow{2}{*}{ Structural alterations/modifications } & Penicillin binding proteins & B-lactam antibiotic \\
\hline & & Cell wall precursors & Vancomycin \\
\hline & Mutations in genes & Ribosomal subunits & Streptomycin \\
\hline & Amino acid substitutions & RNA polymerase & Rifamycin \\
\hline & Methylation & 23S rRNA & Macrolide \\
\hline & Mutation & 23S rRNA & Oxazolidinones \\
\hline Reducedpermeability & Reduced expression/defective & Porins & $\begin{array}{l}\beta \text {-lactams, fluoroquinolones, } \\
\text { aminoglycosides, chloramphenicol }\end{array}$ \\
\hline Target protection & Ribosome protection & Ribosome protection proteins & Tetracycline \\
\hline Efflux & Active extrusion & Membrane proteins & All major antibiotics \\
\hline
\end{tabular}

\section{Molecular Mechanism of Bacterial Resistance}

Bacteria have developed diverse means to circumvent the growth-inhibitory properties of antimicrobial agents. Major mechanisms of bacterial resistance to antimicrobial agents include the following: intrinsic resistance, circumstantial resistance, acquired resistance, biochemical mechanisms of drug resistance drug efflux, biofilm formation, horizontal gene transfer, mutation, reduced permeability, genetics of antibiotic resistance, bacterial persistence, and antibiotic inactivation or modification. These drug resistance mechanisms allow bacteria that harbor these mechanisms to survive, or even to actively grow, in the presence of a given anti-microbial agent.
Furthermore, certain bacterial variants have evolved mechanisms to resist multiple drugs, making such variants recalcitrant to chemotherapy against such bacterial strains that are the causative agents of infection in patients.

\subsection{Intrinsic Resistance}

Is the innate ability of a bacterial species to resist activity of a particular antimicrobial agent through its functional, characteristics. Intrinsic mechanism is those specified by naturally occurring gene found on host chromosome such as beta-lactamase of gram negative bacteria and many multidrug resistance (MDR) efflux system. Efflux systems in gram-positive bacteria always comprise a single polypeptide located in the cytoplasmic membrane. The cell envelope of gram negative bacteria is a major barrier for antibiotics and the 
molecular mechanism of antibiotics resistance consists of the plasma membrane, the periplasm and the outer membrane $(E$. coli has innate resistance to vancomycin). The outer membrane is the major barrier for antibiotics penetrate through porins or by passive diffusion through the outer membrane phospholipids bi-layer. The lipopolysaccharide (LPS) forms another barrier for many antibiotics but poly cationic compounds such as Gentamicin and Colistin are being transported through the outer membrane via interaction with LPS in a process called self-promoted uptake. Efflux pumps of the resistance nodulation cell division (RND) super family are major players in antibiotic resistance of gram-negative bacteria [6].

\subsection{Acquired Resistance}

Changes in bacterial genome through mutation or horizontal gene acquisition, on the other hand, may consequently lead to a change in the nature of proteins expressed by the organism. The bacteria 's ability to not only survive in the presence of antibiotics but also to acquire resistance under antibiotic selective pressures assumes that a threshold concentration of antibiotics is necessary to both induce and sustain resistance genotypes [8].

\subsubsection{Horizontal Gene Transfer}

Transfer of resistance genes from one bacterium to another is called a horizontal gene transfer, the main mechanisms of resistance gene transfer in a bacterium are plasmid transfer, transfer by viral delivery, and transfer of free DNA.Many of the antibiotic resistance genes are carried on Plasmids, transposons or introns that can act as vectors that transfer these genes to other members of the same bacterial species, as well as to bacteria in another genus or species. Horizontal gene transfer may occur via three main mechanisms: transformation, transduction or conjugation. Transformation involves uptake of short fragments of naked DNA by naturally transformable bacteria. Transduction involves transfer of DNA from one bacterium into another via bacteriophages. Conjugation involves transfer of DNA via sexual pilus and requires cell-to-cell contact. DNA fragments that contain resistance genes from resistant donors can then make previously susceptible bacteria express resistance as coded by newly acquired resistance genes) [6].

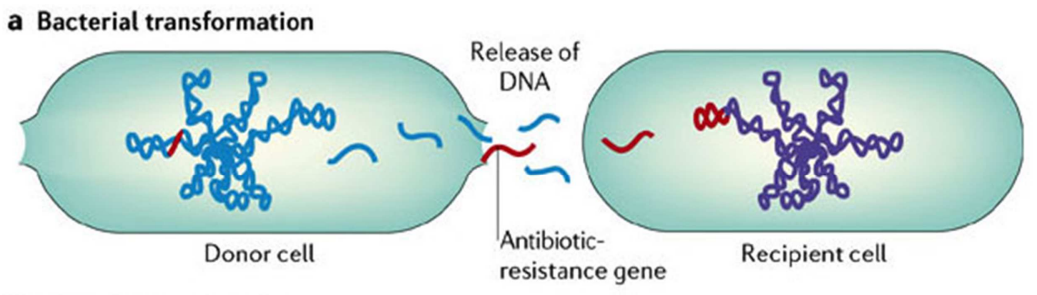

b Bacterial transduction

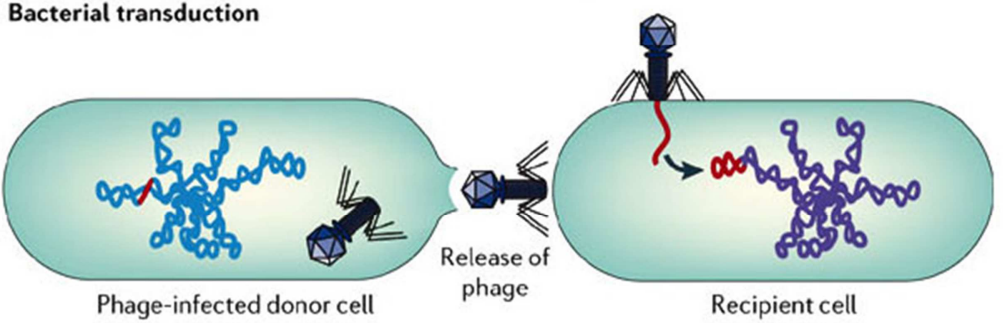

c Bacterial conjugation

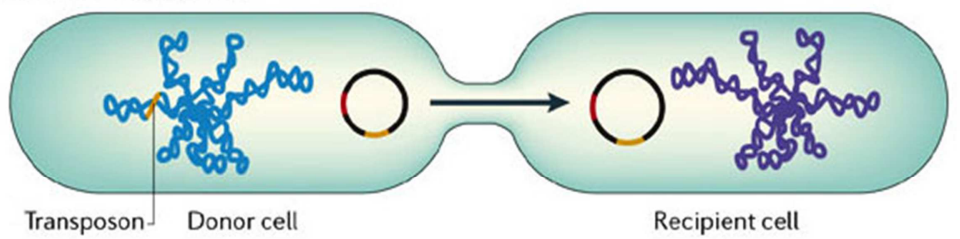

Copyright $(\subseteq 2006$ Nature Publishing Group Nature Reviews | Microbiology

Figure 1.Horizontal gene transfer.

\subsubsection{Mutation}

Is a spontaneous change in the DNA sequence within the gene that may lead to a change in the trait which it codes for. Any change in a single base pair may lead to a corresponding change in one or more of the amino acids for which it codes, which can then change the enzyme or cell structure that consequently changes the affinity or effective activity of the targeted antimicrobials. This increased mutation rate is typically conferred by alterations in the genes that constitute the mismatch repair (MMR) system (mutS, mutL, mutH, mutT, mutY, mutM, and uvrD). Mutations in the MMR system also increases the prevalence of genetic recombination, providing diversity to antibiotic resistance mechanisms [9].

Spontaneous mutations. Chromosomal mutations are quite rare (one in a population of 106-108 microorganisms) and commonly determine resistance to structurally related compounds [1]. These mutations occur as errors of replication or an incorrect repair of damaged DNA are called 
spontaneous mutations or growth-dependent mutations. Resistance to quinolones in E. coli is caused by changes in at least seven amino acids in the gyrAgene or three amino acids in the parCgene [1], whereas only a single point mutation in the rpoBgene is associated with a complete resistance to rifampin [1].

\subsubsection{Reduced Permeability or Uptake}

Acquired resistance to antibiotics through a decrease in the permeability of the cell membrane requires major structural changes in the membrane [6]. Compared with gram-positive species, gram-negative bacteria are intrinsically less permeable to many antibiotics as their outer membrane forms a permeability barrier [6].One mechanism that results in reduced drug permeability in bacteria is the cell wall's lipo polysaccharide (LPS), which consists of lipid A, a core consisting of polysaccharide and O-antigen [11]. Bacteria that harbor LPS moieties show resistance to erythromycin, roxithromycin, clarithromycin and azithromycin in gram-negative bacteria such as strains of Pseudomonas aeruginosaand Salmonella enterica, all of which are serious pathogens, especially in immune-compromised patients [11]. Another mechanism that confers reduced permeability involves the porin channels that reside in the outer membrane and allow small molecular weight molecules, such as antimicrobial agents, to gain cellular entry. Drug resistant bacteria alter the expression of these outer membrane proteins such that they fail to integrate into the outer membrane or are functionally defective, thus preventing the entrance of growth-inhibitory molecules [11]. Clinically important bacterial pathogens like Serratiamarcescens, Salmonella enterica, Klebsiella pneumonia and Pseudomonas. aeruginosa, have utilized this reduced drug uptake system to resist important anti-microbial agents, such as the beta-lactams, fluoroquinolones, aminoglycosides, as well as chloramphenicol [6].

\subsection{Circumstantial Resistance}

Is the difference between the in vitro and in vivo effects of an antimicrobial agent. Agents that appear to be active in the laboratory may be ineffective in vivo because of failure to reach the site of infection, such as the inability of first generation cephalosporins to cross the blood-brain barrier. Drugs such as aminoglycosides may be inactivated; in vivo antagonist of trimethoprim /sulfamethoxazole can be overcome by enterococci via their inability to take up and internalize corporate environmental folate [10].

\subsection{Genetics of Antibiotic Resistance Mechanisms}

Until the 1950s, it was not clear how the bacteria acquire drug resistance. Then, Joshua Lederberg devised replica plating, and demonstrated that the antibiotic resistant mutants are pre-existing. Thus, the antibiotics merely selected these mutants. Then, in 1988, John Cairns showed that when the bacteria are not growing, they are nevertheless able to acquire new mutations, due to some genetic alteration process. Those mutations are called adaptive mutations. It was never formally proven that adaptive mutations cause antibiotic resistance; however, it is possible, particularly in non-growing forms of bacteria. There are different mechanisms of antibiotic drug resistance, which are due to chromosomal mutations:

\subsubsection{Enhanced Efflux.}

One of the most common drug resistance mechanisms is active efflux of drugs from the inside of bacterial cells. Such drug resistant bacteria harbor energy-driven drug efflux pumps which extrude antimicrobial agents thus reducing their intracellular concentrations to sub- or non-inhibitory levels. There are two main types of active efflux pumps. The first type, called primary active transport, uses the hydrolysis of ATP to actively efflux drugs from cells, while the second type, called secondary active transport, uses an ion gradient for active drug efflux from cells. The ATP driven transporters are also known as ABC (for ATP-binding cassette) or P-glycoprotein transporters. Both active transport systems are used by bacteria to resist the inhibitory effects of antimicrobial agents and are often referred to as efflux pumps [7].

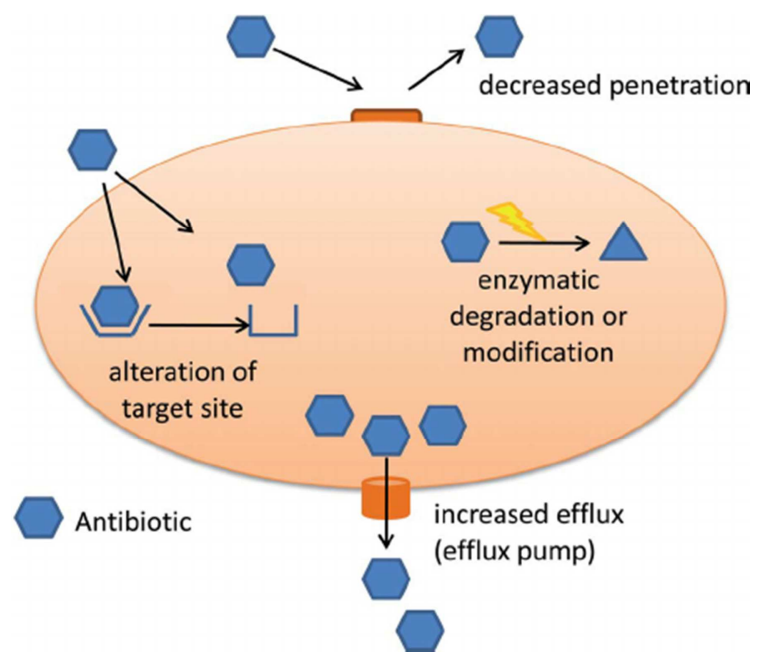

Figure 2.Mechanism of enhanced efflux drug resistance.

\subsubsection{Enzymatic Inactivation}

Drug inactivation or modification: for example, enzymatic deactivation of penicillin $G$ in some penicillin-resistant bacteria through the production of $\beta$-lactamases. The emergence of carbapenem-resistant gram-negative pathogens poses a serious threat to public health worldwide. Klebsiella pneumonia carbapenemases (KPCs) and carbapenemases of the oxacillinase-48 (OXA-48) type have been reported worldwide. New Delhi metallo- $\beta$-lactamase (NDM) carbapenemases were originally identified in Sweden in 2008 and have spread worldwide rapidly [12]. Most commonly, the protective enzymes produced by the bacterial cell will add an acetyl or phosphate group to a specific site on the antibiotic, which will reduce its ability to bind to the bacterial ribosomes and disrupt protein synthesis [12].

\subsubsection{Alteration or Over-Expression of the Drug Target}

Alteration of target- or binding site: for example, alteration of penicillin binding protein (PBP) the binding target site of 
penicillin in methacillin resistance Staphylococcus aureus (MRSA) and other penicillin-resistant bacteria. Another protective mechanism found among bacterial species is ribosomal protection proteins. These proteins protect the bacterial cell from antibiotics that target the cell's ribosomes to inhibit protein synthesis. The mechanism involves the binding of the ribosomal protection proteins to the ribosomes of the bacterial cell, which in turn changes its conformational shape. Altering an, antibiotic's target protein directly at the DNA level is a common mechanism of target modification This allows the ribosomes to continue synthesizing proteins essential to the cell while preventing antibiotics from binding to the ribosome to inhibit protein synthesis [12].

\subsection{Phenotypic Drug Resistance}

Phenotypic drug resistance refers to the fact that when the bacteria are not growing, they can become unsusceptible to antibiotics. Then, when the bacteria are sub-cultured into a fresh media, and they begin to grow again, they regain their antibiotic susceptibility. This complex mechanism has been posing significant problems as in biofilm infections and particularly for TB chemotherapy [13].

\subsubsection{Biofilm Formation}

Biofilms account for a great proportion of all microbial infections in the human body bacterial biofilms are highly resistant to the host's antibodies. In addition, antibiotics that kill planktonic cells often times fail to kill bacterial cells within a biofilm. Among the mechanisms proposed to explain this increased resistance of biofilms to antibiotics is delayed antibiotic diffusion in biofilms [13]. Biofilm is a structured population of bacteria embedded in a matrix, which is composed by polysaccharides, proteins and extracellular DNA Initially, the bacteria simply attach to surfaces irreversibly. Then, early biofilms are formed, and turn into mature biofilms. They are then able to release new organisms off the structure. Biofilm bacteria are extremely resistant to antibiotics. When we compare the susceptibility of the planktonic form and biofilm, we observe that antibiotic imipenem can destroy planktonic organisms of Pseudomonas aeruginosaeffectively at $1(\mu \mathrm{g} / \mathrm{ml})$, but require at least $1024(\mu \mathrm{g} / \mathrm{ml})$ to fight against biofilm. The Biofilm structure is extremely complex. The bacteria are divided into different sub-populations, ranging from an almost spore-like sub-population, to a more actively metabolizing population at the colony surface [6].The contribution of such a cell-density-regulated program to persistence was initially rejected since addition of spent medium containing quorum-sensing molecules could not induce persistence in exponential cultures of $E$. coli, Pseudomonas aeruginosaor Staphylococcus epidermidis. However, later studies found that the quorum-sensing molecules pyocyanin and the acyl-homo serine lactone 3-OC12-HSL increase persister numbers in P.aeruginosawithout affecting persistence in $E$. coli or $S$. aureus. Another quorum-sensing molecule in P. aeruginosa, 2-amino acetophenone, also induces persister formation, most likely by shutting down translation activity.
Furthermore, it induces tolerance in another pathogenic species incapable of producing 2-amino acetophenone like Acinetobacterbaumanniiand Bacillus thailandensis. Pyocyanin produced by $P$. aeruginosaalso shows cross-species induction of persistence in A. baumannnii), which points to the possible existence of more general persistence-inducing mechanisms in natural poly microbial settings [14].

\subsubsection{Salicylate -Induced Antibiotic Resistance}

Another form of phenotypic drug resistance is mediated by salicylic acid, which is the active component in aspirin. Different organisms have been found to have Salicylate-mediated drug resistance. E. coli is the best example, and additional ones include Klebsiella, Pseudomonas, Burkholderia, and also M. tuberculosis. In has been demonstrated that in the presence of Salicylate, Tuberculosis bacterium (TB) is less susceptible to isonicotinic acid hydrazide (INH), Rifampicin, ethambutol (EMB), and para-aminosalicylic acid (PAS). Preliminary experiments in the mouse model of TB demonstrate that aspirin can antagonize the activity of $\mathrm{INH}$, indicating that it might also have some effect in-vivo.

Salicylate binds to MarR in order to release the suppression of the MarAB operon. MarA encodes a transcription factor, which in turn, activates the transcription of the efflux pump acrAB, as well as the membrane channel to $1 \mathrm{C}$, which is required for the functioning of the pump. Thus, the first drug resistance mechanism is conducted through increased efflux. In a second mechanism, MarA enhances the transcription of micF, an anti-sense RNA for ompF, a membrane porin required for entry of antibiotics. Thus, micF shuts down the expression of ompF through antisense. When the porin expression is reduced, the drug intake is reduced as well [15].

\subsubsection{Bacterial Persisters}

'Persistence' can be defined as the presences of transient, phenotypic variants within a susceptible population that survive treatment with lethal doses of antibiotics. Persisters are transiently tolerant variants that allow populations to avoid eradication by antibiotic treatment. Their antibiotic tolerance is non-genetic, not inheritable and results from a phenotypic switch from the normal, sensitive cell type to the tolerant, persister state. At present, persister cells have been identified in almost every bacterial species examined, at levels covering several orders of magnitude, typically between $0.001 \%$ and $1 \%$. Numerous reports exist on persistence in both gram-positive and gram-negative bacteria. In addition, persisters have been described in eukaryotes such as yeast where they show tolerance to antifungals. Even human cancer cell lines are able to form persister-like cells that can survive chemotherapy and have been shown to cause therapy failure and to promote development of drug resistance [14].

\section{a) The Toxin-Antitoxin (TA) Model}

Toxin-antitoxin systems (TAs) are ubiquitous among bacteria and play a crucial role in the dissemination and evolution of antibiotic resistance, such as maintaining 
multi-resistant plasmids and inducing persistence formation. Generally, activities of the toxins are neutralized by their conjugate antitoxins. In contrast, antitoxins are more liable to degrade under specific conditions such as stress, and free active toxins interfere with essential cellular processes including replication, translation and cell-wall synthesis. TAs has also been shown to be responsible for plasmid maintenance, stress management, bacterial persistence and biofilm formation [16].

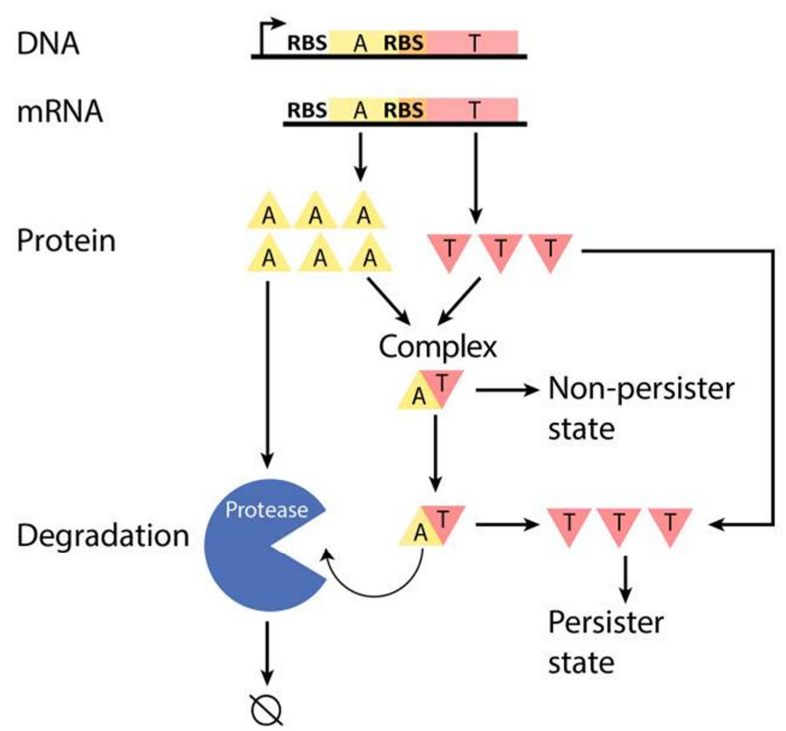

Figure 3.Schematic of a typical Type II toxin-antitoxin (TA) system. A TA system operon is transcribed to produce a corresponding $m R N A$, which is then translated to produce toxin and antitoxin proteins. With sufficient concentration of anti-toxin protein. [17].

\section{b) PhoU}

Recently, a new persisted gene, PhoU, through a transposon based screen in E. coli. The PhoU mutant displays high susceptibility to a range of different antibiotics, such as Ampicillin, Streptomycin, Sulfa drugs, and Quinolone drugs. It is also more susceptible to different conditions, such as heat, starvation, acid $\mathrm{pH}$, and weak acids. The $\mathrm{PhoU}$ mutant phenotypes can be complemented by a wild type PhoU gene. An interesting feature of the PhoU mutant is that it is highly susceptible to Ampicillin in the stationary phase. Many other antibiotics, especially penicillin, are not active against stationary phase bacteria, but merely against growing bacteria. We have also demonstrated, through microarray experiments, that the PhoU mutant has a hyper-active metabolism. Thus, PhoU appears to be a suppressor mechanism for cellular metabolism. When it is expressed, it shuts down cellular metabolism. Although the detailed mechanism is not clear yet, they believe that PhoU could be an interesting drug target for killing persister bacteria. We are therefore currently trying to raise the interest of some pharmaceutical companies in designing drugs targeting PhoU [15].

\section{Conclusions}

Miss and massive usage of antibiotics in clinical practice resulted in resistance of bacteria to antimicrobial agents. Bacteria use varies resistance mechanisms to protect themselves. Acquired resistance arises from mutations, gene transfer by conjugation or transformation, transposons, and bacteriophages. The following types of resistance mechanisms are used by bacteria: antibiotic inactivation, target modification, altered permeability, and also different phenotypic mechanism used to resist antibiotic. It is necessary to determine bacterial resistance to antibiotics of all classes (phenotypes) and mutations that are responsible for bacterial resistance to antibiotics (genetic analysis). Better understanding of mechanisms of antibiotic resistance, location of genes in a chromosome and their expression would allow us to develop screening and control strategies that are needed to reduce the spread of resistant bacteria and their evolution.

\section{Recommendations}

Studies of resistance mechanisms of resistance must be a compulsory requirement at an early stage of drug development; such studies will advise academic institutions and industry to work together. Strategies towards treatment options should be developed to target on virulence factors of pathogensinstead of whole bacteria, for example, develop drugs that target the plasmids containing resistance genes become or drugs that target the adhesion of virulent bacteria totissue and promote appropriate drug use policies and public health education on appropriate antibiotic drug use.

\section{References}

[1] Agnè, G., Astra, V., Rima, N., and Alvydas, P. (2011). Antibiotic Resistance Mechanisms of Clinically Important Bacteria.Medicina (Kaunas) 47(3):137-46.

[2] World Health Organization (2016). Race against time to develop new antibiotics.

[3] Liu, Y.Y, Wang, Y., and Walsh, TR. (2016). Emergence of plasmid mediated colistin resistance mechanism MCR-1 inanimalsandhumanbeings in China. Lancet Infect Dis. 16: 161-68.

[4] O’Neill Review in to AntibioticResistance, March.2017.

[5] Hauser, AR., Mecsas,J. and,Moir, DT.(2016). Beyond Antibiotics: New Therapeutic Approaches for Bacterial Infections. Clin Infect Dis. 63: 89-95.

[6] EngidawAbebe, BetelihemTegegne and Solomon Tibebu (2016). A Review on Molecular Mechanisms of Bacterial Resistance to Antibiotic. European Journal of Applied Sciences 8 (5): 301-310.

[7] Sanath Kumar and Manuel F.Varela (2013). Molecular Mechanisms of Bacterial Resistance to Anti-Microbial Agents. Science, technology and education.

[8] Meredith Schroeder, Benjamin, D. Brooks and Amanda, E. Brooks (2017). The Complex Relationship between Virulence and Antibiotic Resistance. Review 2017. 
[9] Rodríguez-Rojas, A.; Rodríguez-Beltrán, J.; Couce, A.; Blázquez, J.(2013). Antibiotics and antibiotic resistance:A bitter fight against evolution. Int. J. Med. Microbiol303: 293297.

[10] Olowe O Adekunle (2012). Mechanisms of Antimicrobial Resistance In Bacteria, General Approach. International journal pharma medicine and biological science 1(2): 22785221.

[11] Monack, D.M. (2012). Salmonella persistence and transmission strategies. Curr. Opin. Microbio. 15: 100-107.

[12] Lee, C.R., Lee, J.H., Park,K.S., Kim,Y.B., Jeong,B.C., Lee,S.H (2016). Global dissemination of carbapenemase producing Klebssiella pneumonia. Microbial 7:895.

[13] Tarek M. Mahfouz and Michael J. Young (2017). New Bacterial Targets and Computational Methods against Bacterial Resistance. Medical Research Archives 5: 4.
[14] Bram Van den Bergh, Maarten Fauvart and Jan Michiels (2017). Formation, physiology, ecology, evolution and clinical importance of bacterial persisters. FEMS Microbiology Reviews 41: 219-251.

[15] Ying Zhang (2007). Mechanisms of Antibiotic Resistance in the Microbial World. Clin. Pharmacol. 82:595-600.

[16] Qiu E. yang, Timothy R. Walsh (2017). Toxin-antitoxin systems and their role in disseminating and maintaining antimicrobial resistance. FEMS Microbiology Reviews41: 343-353.

[17] Heather S. Deter, Roderick V. Jensen, William H. Mather, and Nicholas C. (2017) Mechanisms for Differential Protein Production in Toxin-Antitoxin Systems. Toxins9(7): 211. 